\title{
"Piece by Pieces": Interactive Art Contents via Web Technology
}

\author{
Chan $\operatorname{Lim}^{1}$ and Hyeyoung Yoo ${ }^{2}$ \\ ${ }^{1}$ Department of Global Media, Soongsil University \\ ${ }^{2}$ Department of Art \& Technology Graduate School of Advanced Imaging Science, \\ Multimedia and Film, Chung-Ang University \\ chanlim@ssu.ac.kr; hyy345@gmail.com
}

\begin{abstract}
Through various media, including the Internet, our lives projected to represent the interaction and can be recognized. Nestled in the heart of individuals is no longer a thought to influence the behavior of individuals, not only, to project his mind to communicate with the device via a completely different person to determin the behavior of the new.

In this work, these individuals have projected their minds, which consists of several pieces Keyword own pieces together through the new process of visualizing yourself in the show.

Moreover, simply to visualize their appearance, as well as the reflection of the camera for their appearance are added to interactive ements, theicappearance according to the movement becomes more finely, expressed as number of piees in the process will show the.

Using this process by presenting webArt, we ath cherish, but his mind was, perhaps insignificant and they do not even see it in the window of his thoughts scattered slick new world to discover his passion for life and motivation will receive.
\end{abstract}

Key Words: Interactive arts, Combination with the parts, Web Art

\section{Introduction}

Through the process of contmuous thinking and communicating with one another, we have finally been finding ourselves somehow. Before various methods of internet based media came along, we only had limited expressive ways of personal inner thoughts stems from body itself to verbal and written and so on. However, in terms of processing speed dealing with information system, one second is like one inch when information technology is trying to divide one nanometer into something closer to void. Nowadays, cyber space is not simply a virtual space but an abstract physical territory where an individual shares and communicates his or her ideas through various devises. Any individual has a certain image of something represents an phenomenal or psychological matters on search engines, an individual could easily match the thought of something meaningful with the specific images which have been floating around him or her. Most major search engines have key word input window on their UI interface and we usually name it 'key word' window. Once you punch in a certain familiar keyword that you are definitely familiar with, you would have a series of images pretty much predictable. However, besides of your pre-visualized predictable images, you could also find millions of images that you had not expected from once your familiar images from your own definite the 'key word'. More than that, qualities and quantities of images generated from the keyword keep popping up. Individuals could finally experience how limited I was. Images are not beings but becomings. Even images are obtaining new meanings in real time. Your ideal image from your positive keyword could be interpreted as opposite and vice versa. Someone else thoughts were never accepted by different social, political and 
religious issues could widen visions once were thought to be changeless. Images are limitless and timeless through human races, genders, and ages. And experiencing differently being utilized others images of something commonly being used words is amazing ways of understand and respect others value and makes society healthy. Along with your image from a single 'keyword', observing others images keeping on juxtaposing stemming from the same 'keyword' on real time is the process of reconsidering yourself and the world around. I type in a keyword on the search engine and the web shows the numerous images. There are many of my representatives and many are not. I respect "many are nots" more than "many of me" and they are becoming part of me as a tool to communicate with others. the result would reflect back to me as a new becoming me and the pursuit of this paper.

\subsection{Purpose of the Work}

Alois riegl told that progress of art history is only being taken by 'wollen'(the acting of will) not simply by 'konnen'(thing thing you can). However, the flows of an from Egypt to modern age, have been taken into consideration with 'konnen in terms of technology which make 'wollen' into 'konne'. In other words progress of art history should be understood with art technology of its own period. Cave men found blood of dead animal and mud their artistic medium. Canvas paper and color material took its place and they no longer needed cave wall to display. With computer generated information technology have been updating, WWW have been replacing canvas with almost limitless capacity of distance and efficiency. Once only selected upper-class had all the privileged opportunities to anticipate the art related affairs but everyone becomes gallery, commentator and critiques along with information technologies. Among those, internet has been being practical since relatively not long ago in the flow of art history. People might have assumption that media related ar is somehow hard to reach; highlyconceptual, philosophical, and nol amusing cetc. however, since web art introduced, people have more access towards media art in many genres and even participating within the contents due to the internet. This could mean that people almost have limitless access to the artists and their works communicating mutually without physical barriers. Among many media technologies, Internet, html, and Flash are playing major roles which have brought people into web art. Different from html and web technology, flash should be categorized as software developing tool. However its intensive efficiency and usage, from the year 2000 to nowadays flash is one of the most powerful web technologies continually overgrowing its multi-media capacity widely from web page to sound, animation and videostreaming. That is because initiating period of web and html had only oriented 'document' and 'hyper link' and they had never considered multi-media supporting such as sound, video and animation. With efficient compatibility which web browser which html and web lacks, flash have been widening its multi-media function along with web. Not only web page, SW and plug-ins but flash have been playing a major tool to create media related art. Technically, almost web art is heavily relying on flash due to the in erchangeability with web which html is not. My recent work in this paper is flash and html based to emphasize since the work is heavily rely on multi-media and animation.

\section{Workflow}

\subsection{Concept of Web Art}

Simply speaking, the term WebArt is an artistic activity appearing on on-line, net wok and internet web browser. Previously used artistic medium such as canvas has gone beyond its physical limits. Virtual space on internet especially web browser oriented artistic performance has been taking canvas. However, this explanation of the term WebArt somehow limited and too vague to include other possibilities. Below is how Wikipedia identifies WebArt. "Internet art( often referred to as net art) is a form of digital 
artwork distributed via the internet. This form of art has circumvented the traditional dominance of the gallery and museum system delivering aesthetic experiences via the internet. In many cases, the viewer is drawn into some kind of interaction with the work of art. Artists working in this manner are sometimes referred to as net artists. Internet art can happen outside the technical structure of the internet, such as when artists use specific social or cultural internet traditions in a project outside of it. Internet art is often - but not always - interactive, participatory, and multimedia-based. Internet art can be used to spread a message, either political or social, using human interactions. The term internet art typically does not refer to art that has been simply digitized and uploaded to be viewable over the internet. This can be done through a web browser, such as images of paintings uploaded for viewing in an online gallery. Rather thins genre relies intrinsically on the internet to exist, taking advantage of such aspects as an interactive interface and connectivity to multiple social and economic cultures and micro-cultures. It refers to the internet as a whole, not only to web-based works. Theorist and curator Jon Ippolito defines "ten myths" about internet art in 2002. He cites the above stipulations, as well as defining it as distinct from commercial web design, and touching on issues of pernanence, achievability, and collecting in a fluid medium.

Take another look on WebArt definition for further understanding There are largely two different points of views when the viewer is taking WebArt into consideration. Firstly WebArt could be defined as 'art on web'. However, if you follow this definition, it is not so different from visiting museums or galleries on the virtual space through navigating on- line network. This virtual tour is merely on-line catalogue which is digitized so you might refer it as to "digital museum or gallery WebArt co@ld be declared only when the art is created utilizing web technology (art based on web). (n other words, WebArt is an artistic creation displayed on web browser Web art st group leader Joongjae Lee says "virtual space must be the field of artistie creating and the contents should be produced and distributed videos, sound and image through internet." Webby awards winner Younghae Chang Industries has shown the standard Gdentification of WebArt mentioned above. Despite all the efforts trying to set a definition of WebArt, the term is still vague among the viewers. 2013 IDAF: InernationalDigital Art Festival have categorized 'WebArt' and "NetArt' under digitahar boundary indicating that the terminology WebArt could be temporary and cotld have precise identification someday. Therefore, it is safe to say the concept of WebArt as artistic activity distributed via the internet delivering aesthetic experiences through the internet. But this paper is dealing with internet browser based WebArt work not networking based - visualizing how an individual's inner thoughts comes together routed from the same signifier. This art work is called "piece of pieces" and the appearance and technology is limited only using web browser. 


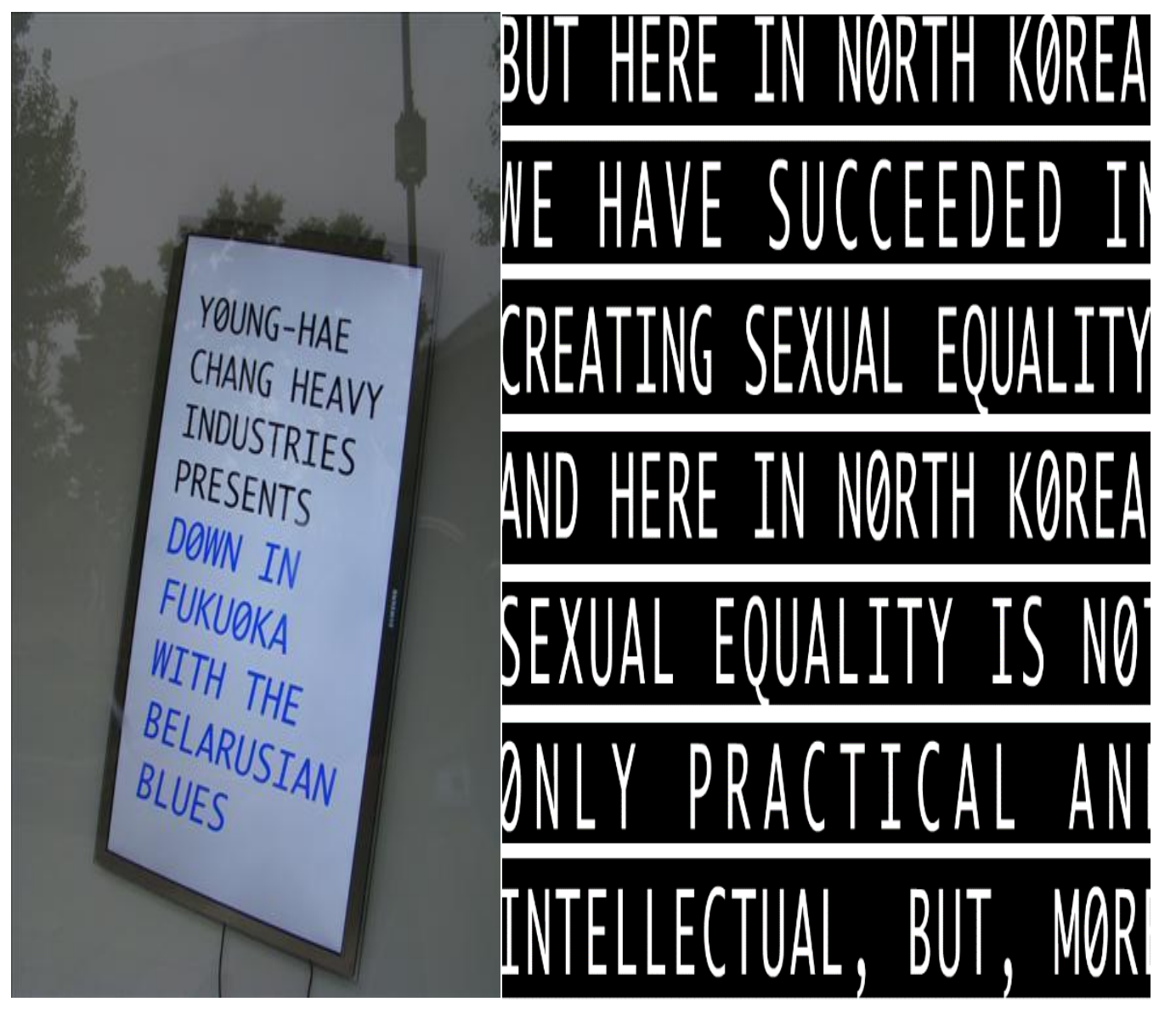

Figure 1. Younghae Chang Heavy Industries

Rapidly developing information technology brightly shines in numerous aspects of our lives and the usage of this information varied by each individual. Internet users want to obtain information of their own interests by punching in keywords in the search engine window. Processed keywords Would be displayed in various formats such as text, images and unpredictable personal thoughts and so on. Countless keywords are continually appearing and disappeating overnight. A certain keyword stays for a while is the index that shares interests of others. They are often ranked in numbers determined by participants' popularity,

In the face of information nestled in the world through visualization, our choice is instinct and simple. Simply information and the word chosen by ourselves need to be understood and comprehended. Such information seeks for another information and topic related toitself. Namely, information needs to be immersed in a topic.

Fon this reason, this paper, piece of you, brings into introspection through important keyord, various search activity, interaction between works and audiences.

However, about works requiring too hard interaction audiences may have resistance, and then this may cause side effect. So we need interaction which is simple and brings audiences pleasure.

Existing media arts are too hard to understand. Of course, though author's style which is profound, various thoughts is not trouble, this may confuse audiences because authors sometimes make a big thing out of it and the meaning of the work is uncertain. So this work enables audiences to instantly comprehend by simple explanations. Furthermore, they can give play to their imagination.

In the old times, by not that they wait and see about art works such as statue, sculpture, but that they search and they move to some places which provide us with good 
information through buttons of 'piece of you', we will always get the opportunity to become an author.

\subsection{Existing Current Works Analysis and Market}

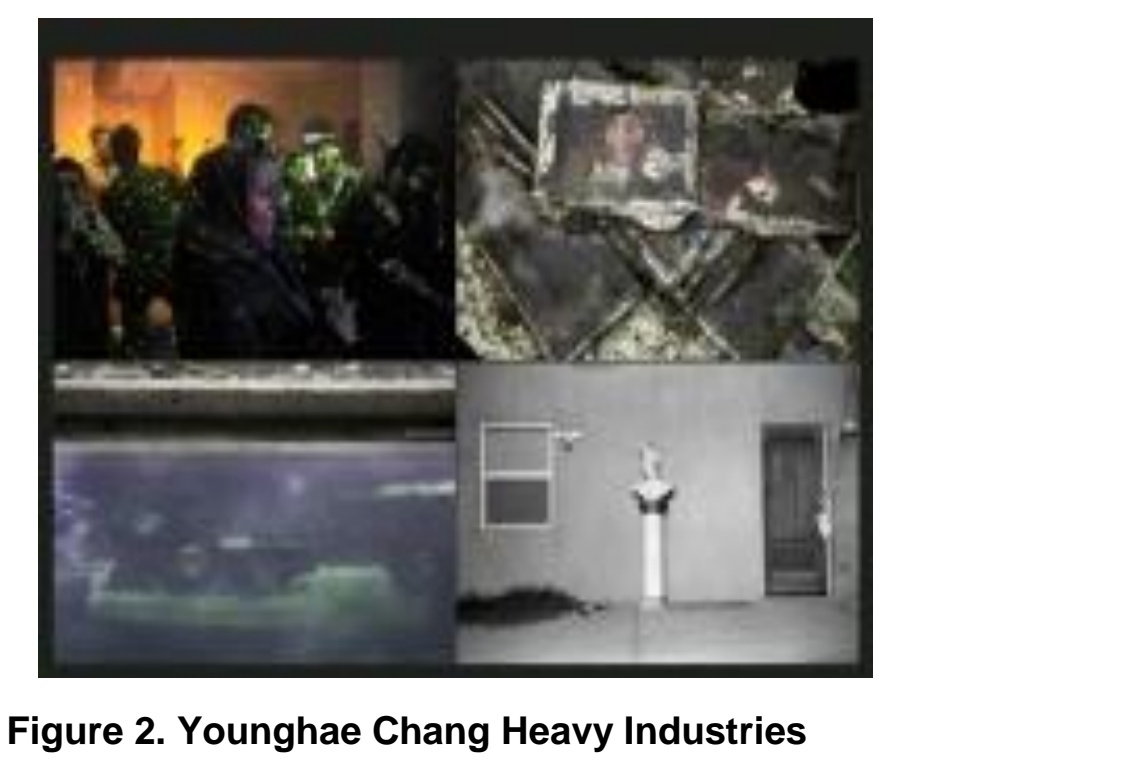

As I mentioned above, behaviors $\triangleleft$ which audiences appreciate the works, not is surprising, but need to exchange interaction among works and viewers. So, the meanings of the media art culture are communcation and comprehension through works.. Informative guide of media arts are countless, but among other things, more impressive, user-oriented and apprehensible works were adopted.

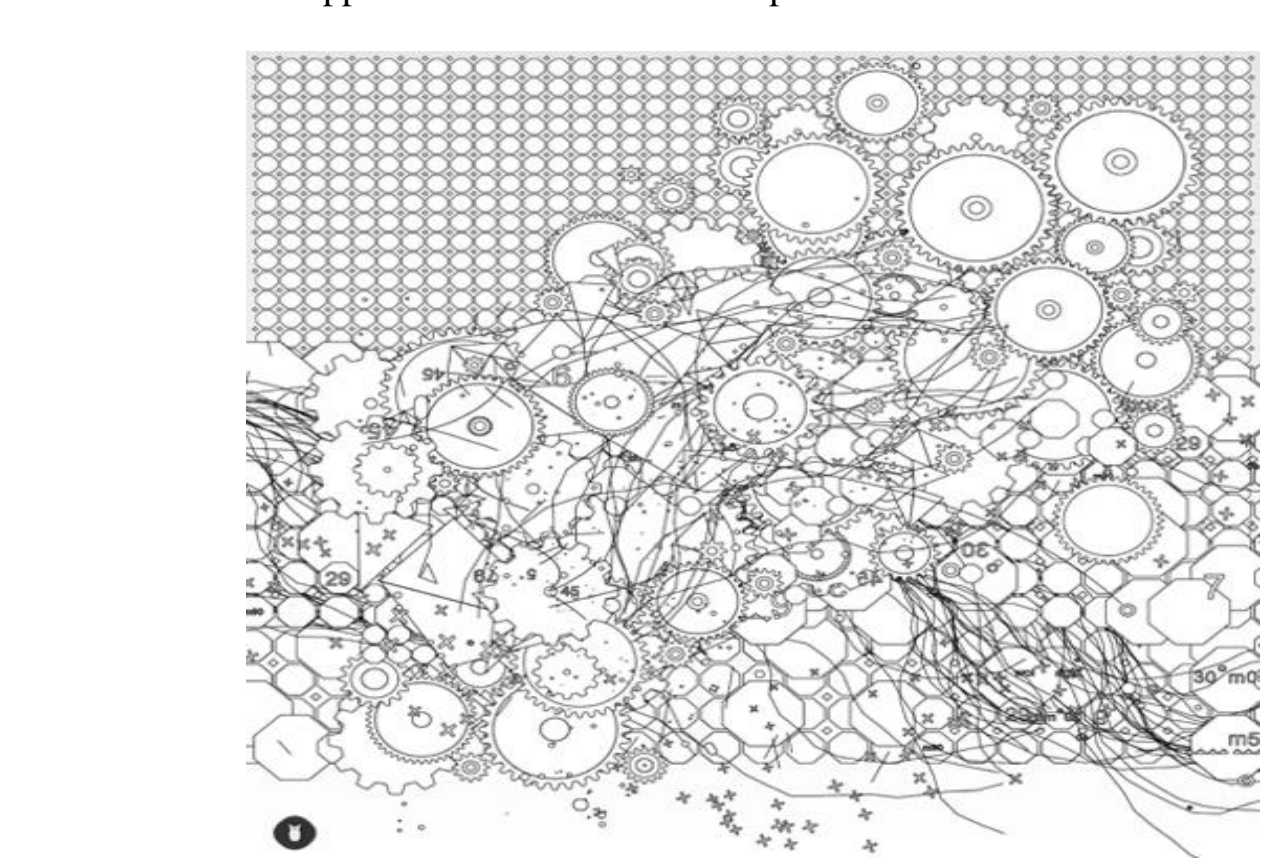

Figure 3. Joshua Davis' NetArt Work 


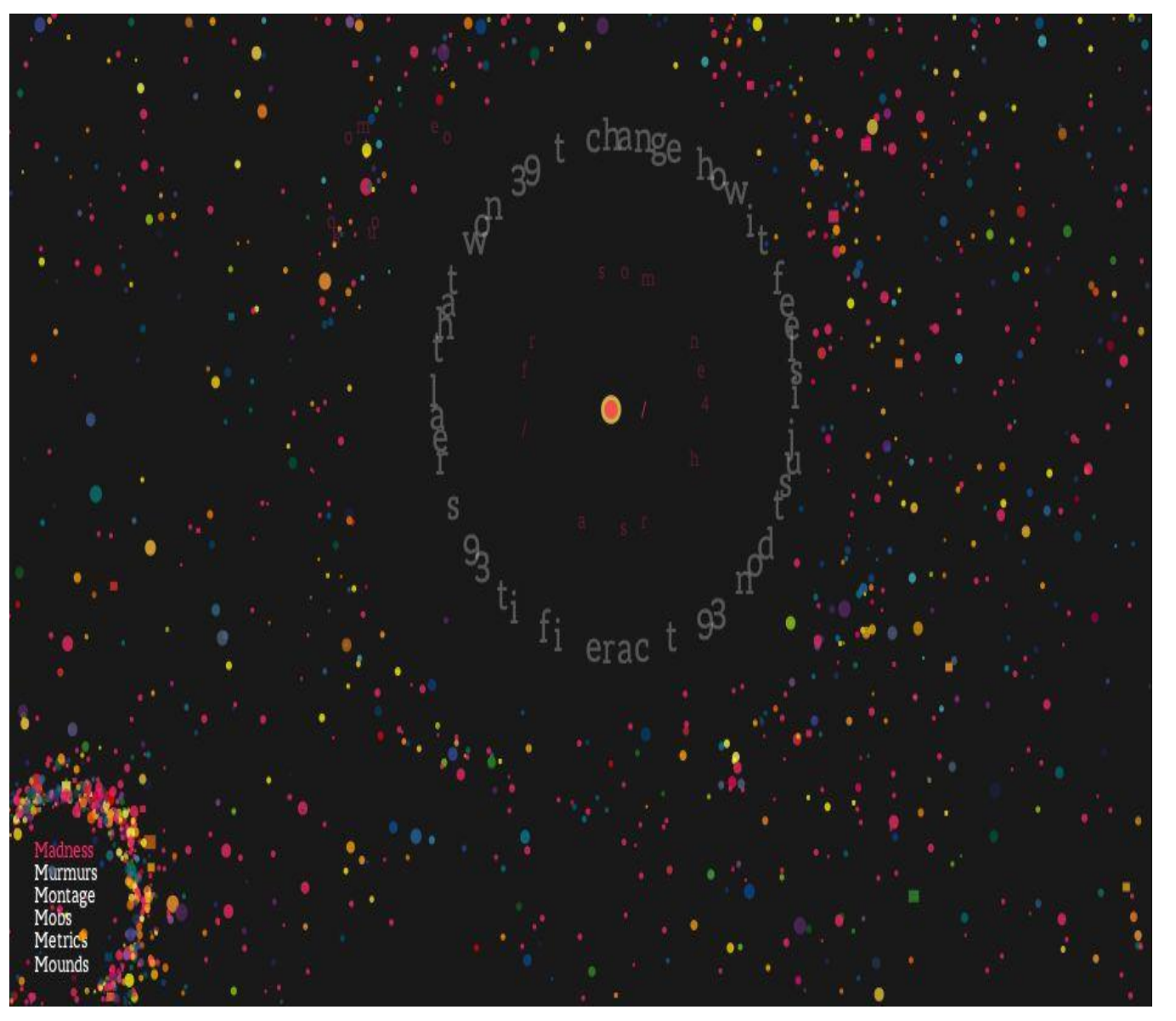

Figure 4. Jonathan Harris "we fegl fine" www.wefeelfine.org

Magnum Photos consists of world - famous media photographer. The homepage of Magnum, Magnum in motion, provides us with general public relations and information and simultaneously is cyber places that display their works. Visitors can choose authors and topics they want from a show listing. And each work is displayed through picture works combined with a series of image, sound, and text. The image control from the project is practicable, which also compresses video - streaming. Without real media, window media, everyone can setup the videos because Flash technique was used in many parts. Joshua Davis, pioneer about art activities through Action Script Programming, attracts public attention, apart from commercial graphic design, due to experimental works. Davis is famous for the starter of art combined with computers known by Dynamic Abstraction. His works operated with technologies, and computers can be appreciated at once upon - a forest.com, or PlayStation (www. playstation.com). At 'http./Www.joshuadavis.com' is an experimental part called "Lab". The works viewed at the part not for a special concept or massage passing, seems exist but for a small game between an author and audiences. "We Feel Fine" produced by Jonathan Harris known for Yahoo! Time Capsule was designed in order to help human beings to understand each other through marks left ,intentionally or not, at the Web.

\section{3. "Piece by Pieces"}

\subsubsection{Process}

Naver search engine's API(sets of technologies that enable websites to interact with each other by using REST, SOAP, JavaScript and other web technologies) is the 
fundamental device of "piece by pieces". Through the search window generated by Naver, "piece by pieces" collects all the images matching to keywords in real time and have them in the shape of photo mosaics. If searched images are not enough to create a certain structure, the same image would appear repeatedly to fill the interface. In this case, the concept of work would not satisfy as originally planned that is communicating and corresponding and finally interacting between the author and the audience. So matching numbers of images from keyword must have at least 100 in order to create dynamic mosaics. To visualize photo mosaic from the searched images from a keyword, on RGB color mode, collected image file is divided into 9 sections. Each section is calculated which color has more values among RGB. Whatever colors less in RGB would be substituted to which has large values. Self-image through web camera is processed the function. Closer color to skin would be chosen to structure and the resolution is presenting $1900 * 2080$ pixels. In order to output visual information, visual recognition algorithm programming is processing the self-image through Flash. Also client - server programming enables collecting images from the keyword through Naver API search engine. Finally, the moving image from the camera-captures image, self-image in the work, is transforming a certain structure with the image from the keyword that you have typed in. you are about to encounter with your self-filled with millions of inages from the keyword you have just entered.

\subsubsection{How "Piece by Pieces" Works}

1) Putting a keyword in the search window on Piece of pieces" UI.

2) Naver image searching engine will bring more than at least 100 matching images from the keyword.

3) After image loading is completed, self-1) nage captured from web-cam will be appearing 4 pieces of mosaic image.

4) The more gestures you make in front of the camera, the smaller the mosaic divides into $1920 * 1080$. So, the user could clearly identify his or her image composited by pixelated mosaics.

5) After a while, when the user stops movung stationary, the numbers of mosaic pixels getting smaller drastically to 4 just like when it had begun.

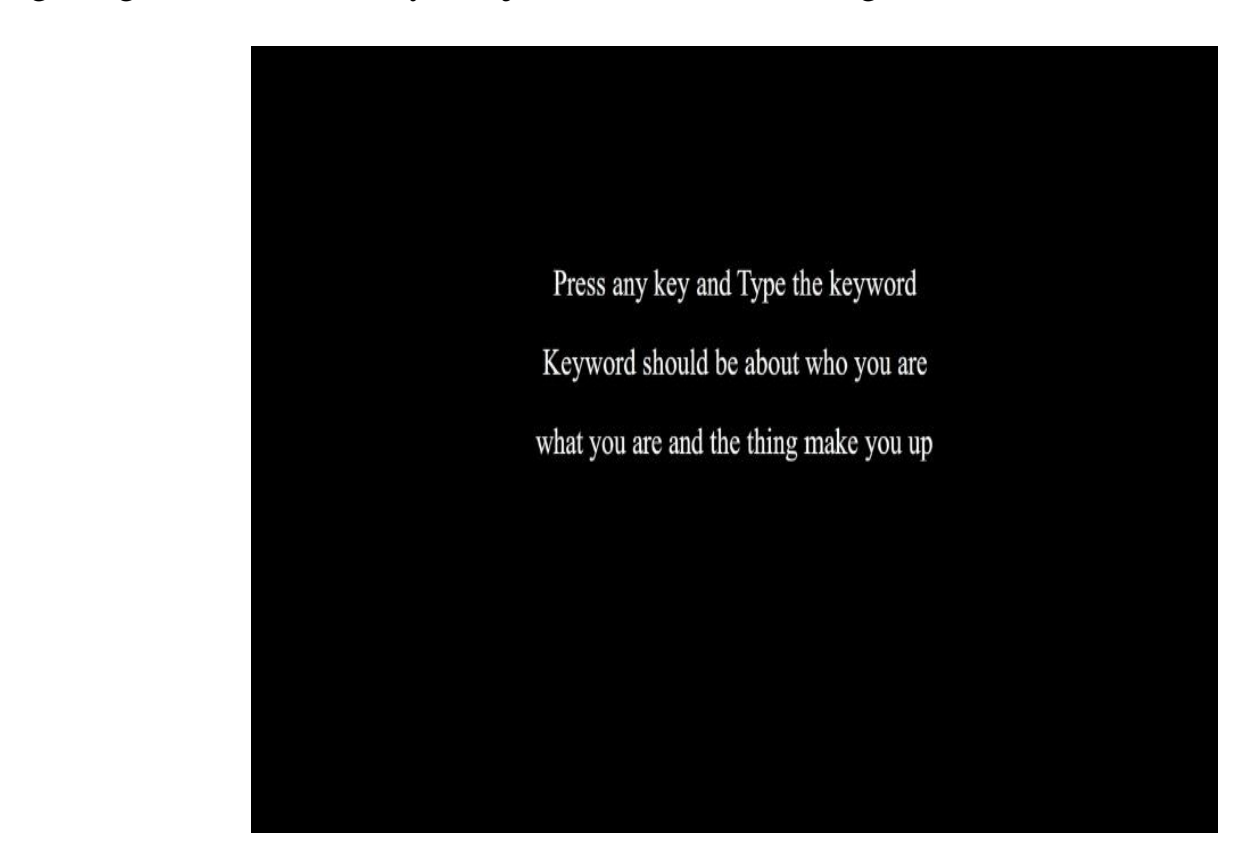


Press any key and Type the keyword

Keyword should be about who you are

what you are and the thing make you up

\section{red}

Figure 5. Keyword Window and Instruction

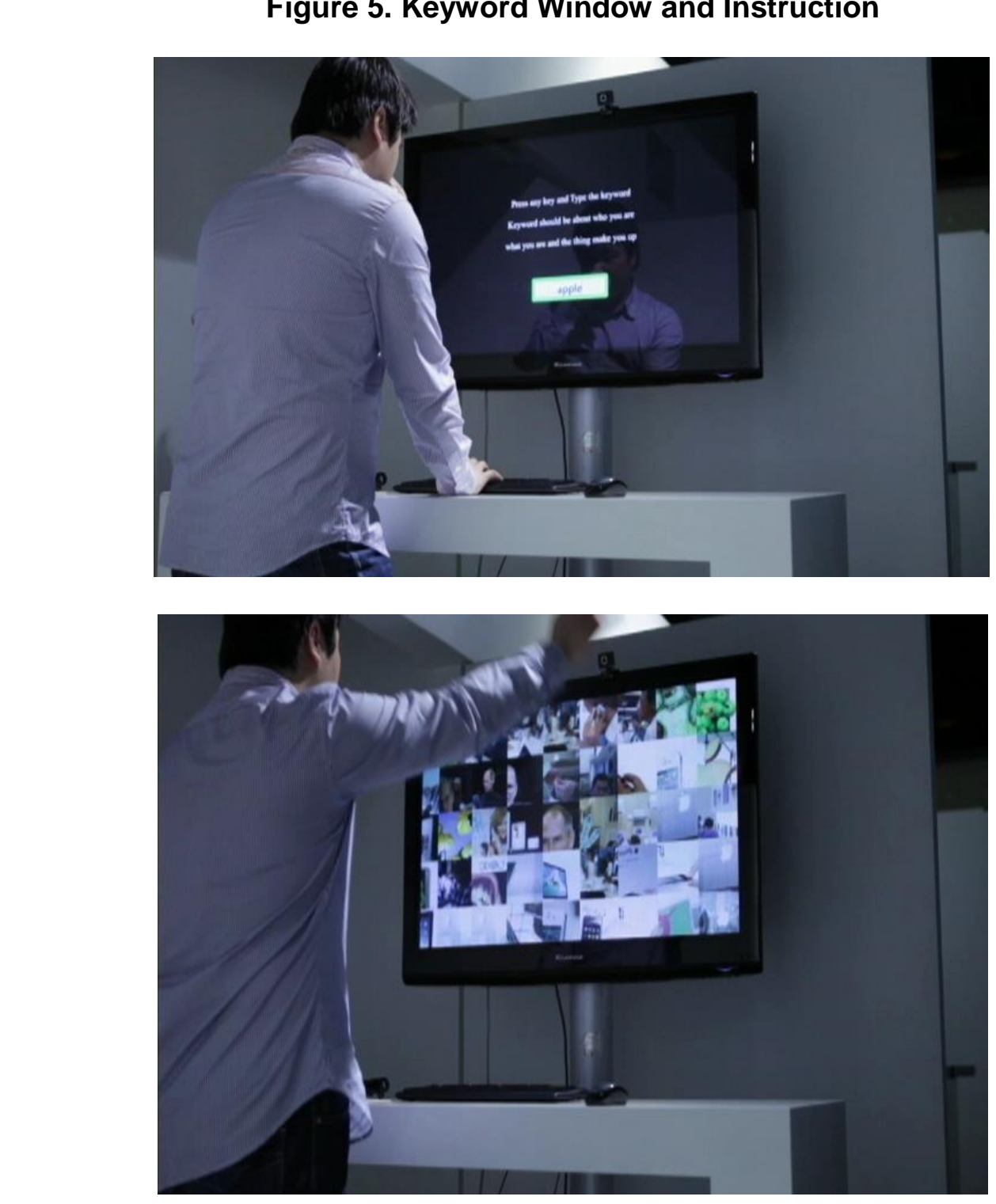

Figure 6. Keyword Matching Images Start to Appear from 4 Mosaics 


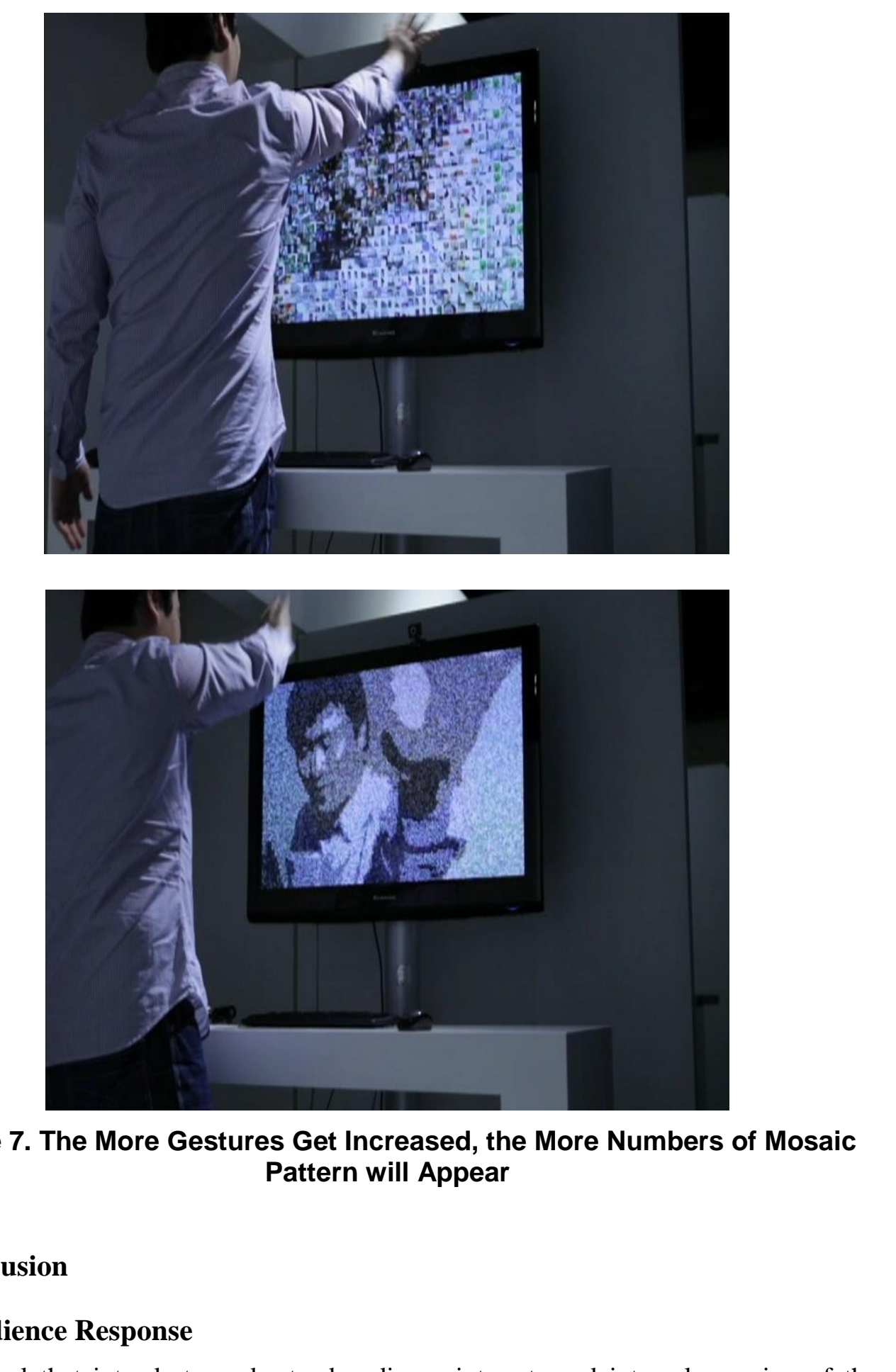

Figure 7. The More Gestures Get Increased, the More Numbers of Mosaic

\section{Conclusion}

\subsection{Audience Response}

The goal that intends to understand audience interests and internal meaning of the works and that means to make media art easy to manipulate has been achieved. Actually after through their own keyword the audiences saw their images drawn, amazing reactions are much part, the shoot photograph service in CAM which immediately was offered was well received by audiences.

Though few audiences intended to understand the deeper meaning of the works, of course, whenever they move, they seem to be surprised, because photo mosaic dynamics are changed at any minute of the day or night. Consequently, most audiences are much interested in works which can come in many ways rather than ambiguous works which 
have profound meaning, and prefer the works which can immediately obtain definite output by their choice and behavior.

\subsection{Improvements Needed for Further Use}

First, it is necessary to change a programming language. Flash is familiar, easy to manipulate, and has been used, to make works requiring dynamic animations and screen handling. Because Flash is an idealized language. It has been used for 3 years.. However, Web programming languages which are essential to make WebArt are ahead of time. Instead of Flash, html5 could be better alternative. Since html 5 has overgrowing advantages of interchangeability with other platforms. With stable data process capacity, html5 is the most flexible and powerful programming language for creating WebArt. Html5 is now able to produce multi-media such as sound, text, image oriented object, video streaming, and interaction along with interaction independently without supporting Flash.

Secondly, "piece by pieces" could be uploaded to web application and shared via social media such as Facebook to communicate with randomly of dered people for vivid interaction in real time. By doing this, users could obtain freedom from desktop computers checking others responses spontaneousty as well

Third, "piece by pieces" generate images from camea input device jnto mosaics in real time. If resolution of each image is different, algorithms must optimized to calculate the differences for adequate result. Otherwise, noticeable delay could happen in-between images due to repeate alculation of resolution of searched images. To avoid the problem, I suggest to consider equipping 'Github'( code management for open source software) to maintain jts best qualities .

\section{Acknowledgments}

This research was supported by the $C_{1}$ ational Research Foundation (NRF: 201317221101).

\section{References}

[1] T. Dreher, "History of Computer Art Chapter VI:Networks, Participation, Hypertext.

[2] B. Schneider, From Clubs to Affinity: The Decentralization of Art on the Internet «491, (2011).

[3] R. Ascott, "Telematic Embrace; visionary theories of art, technology and consciousness", Berkeley: University of California Press, (2003).

[4] B. Rost, "Twilight zone of proteinr sequence alignments", Protein Eng., vol. 12, (1999), pp. 85-94.

[5] T. Corby, "Network Art:P Pactices and Positions", (2006); Routledge.

[6] B. Schneider, "From Clubs to Affinity: The Decentralization of Art on the Internet « 491", (2011).

[7] M. Flanagan, "Navigating the narrative in space: gender and spatiality in virtual worlds", Art Journal, New York: The College Att Association, (2011).

[8] P. Parsons and K. Sedig, "Adjustable properties of visual representations: Improving the quality of humaninformation interaction", Journal of the American Society of Information Science and Technology, vol. 65, no. 3, (2000), pp. 455-482.

[9] D. Daniels and G. Reisinger, "Net pioneers 1.0: Contextualizing early net-based art", Berlin: Sternberg Press, (2009).

[10] K. Sedig, P. Parsons and A. Babanski, "Towards a characterization of interactivity in visual analytics", Journal of Multimedia Processing and Technologies, Special Issue on Theory and Application of Visual Analytics, vol. 3, no. 1, (2012), pp. 12-28.

[11] S. Kiousis, "Interactivity: A concept explication", New Media \& Society, vol. 4, no. 3, (2002), pp. 355-383.

[12] R. Rada and A. Michailidis, "Interactive media", (1995), p. 12.

*Corresponding author: Hyeyoung Yoo, Ph.D Candidate. 
Department of Art \& Technology

Graduate School of Advanced Imaging Science, Multimedia and Film,

Chung-Ang University,

Data+ Lab B217, 301 Art Center Chung-Ang University 221, Heukseok-dong, Dongjak-

gu, Seoul 156-756, Korea

E-mail: hyy345@gmail.com

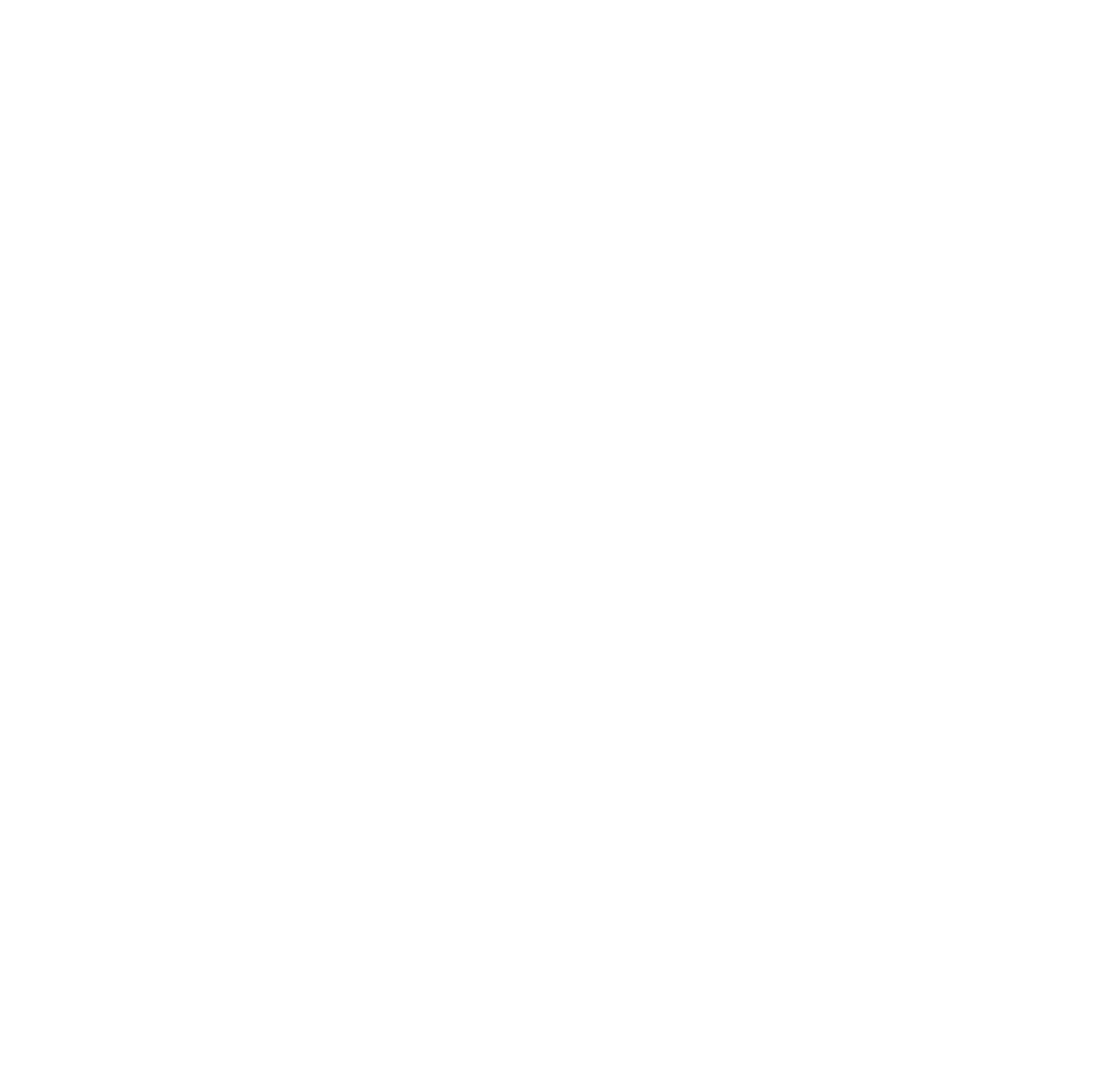


International Journal of Multimedia and Ubiquitous Engineering

Vol. 9, No. 11 (2014)

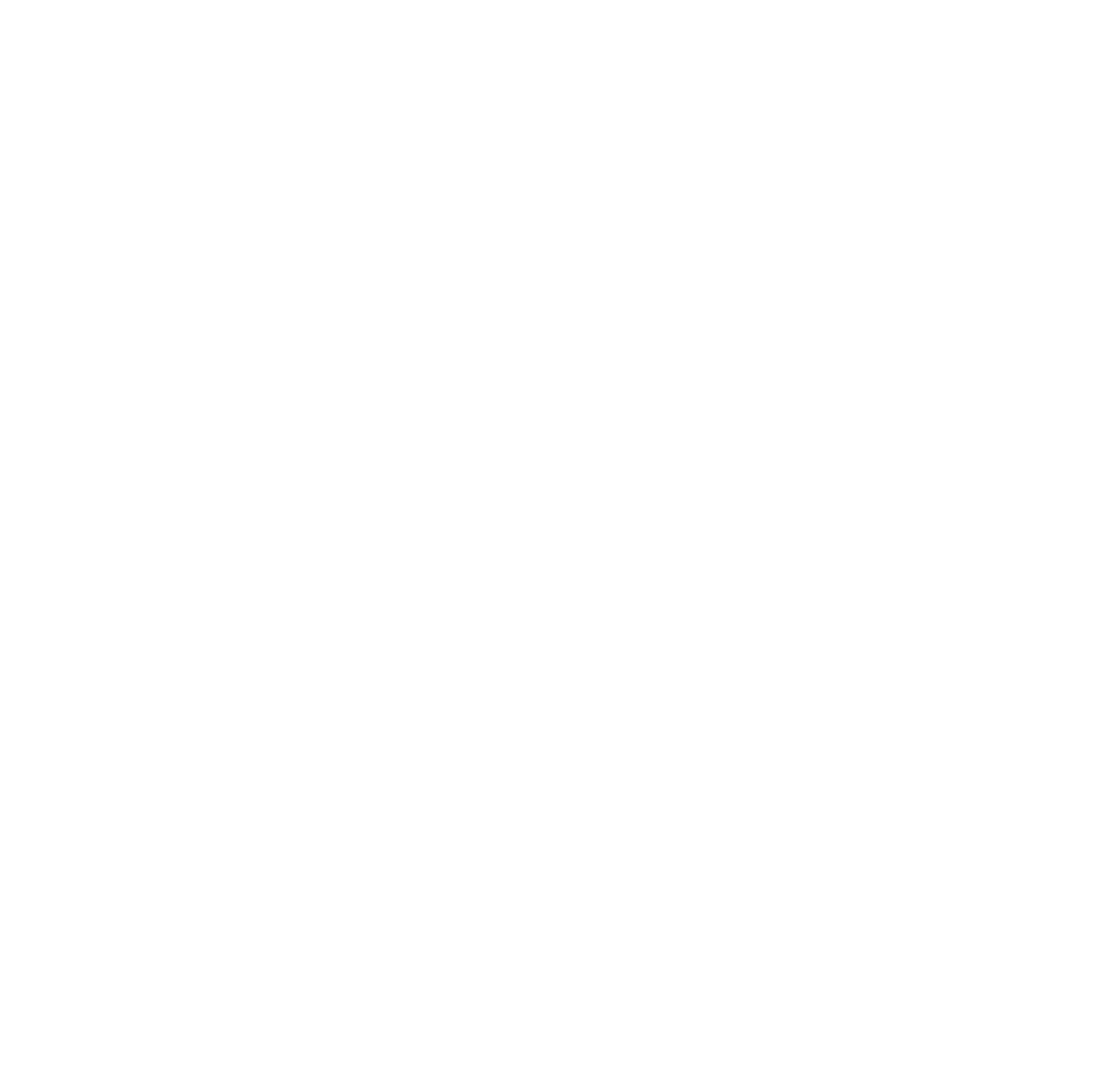

